\title{
Convolution Neural Network Models for Acute Leukemia Diagnosis
}

\author{
Maíla Claro ${ }^{1}$, Luis Vogado ${ }^{1}$, Rodrigo Veras ${ }^{1}$, André Santana ${ }^{1}$, João Tavares ${ }^{2}$, Justino Santos ${ }^{3}$, Vinicius Machado ${ }^{1}$ \\ ${ }^{1}$ Departamento de Computação, Universidade Federal do Piauí - Teresina, Brazil \\ ${ }^{2}$ Instituto de Ciência e Inovação em Engenharia Mecânica e Engenharia Industrial, \\ Departamento de Engenharia Mecânica, Faculdade de Engenharia, Universidade do Porto - Porto, Portugal \\ ${ }^{3}$ Departamento de Informática, Instituto Federal do Piauí - São Raimundo Nonato, Brazil \\ \{claromaila,lhvogado,justinoduarte\}@gmail.com,\{rveras,andremacedo,vinicius\}@ufpi.edu.br, tavares@fe.up.pt
}

\begin{abstract}
Acute leukemia is a cancer-related to a bone marrow abnormality. It is more common in children and young adults. This type of leukemia generates unusual cell growth in a short period, requiring a quick start of treatment. Acute Lymphoid Leukemia (ALL) and Acute Myeloid Leukemia (AML) are the main responsible for deaths caused by this cancer. The classification of these two leukemia types on blood slide images is a vital process of and automatic system that can assist doctors in the selection of appropriate treatment. This work presents a convolutional neural networks (CNNs) architecture capable of differentiating blood slides with ALL, AML and Healthy Blood Slides (HBS). The experiments were performed using 16 datasets with 2,415 images, and the accuracy of $97.18 \%$ and a precision of $97.23 \%$ were achieved. The proposed model results were compared with the results obtained by the state of the art methods, including also based on CNNs.
\end{abstract}

Index Terms-leukemia diagnosis, convolutional neural network, computer aided diagnosis.

\section{INTRODUCTION}

The bone marrow produces a large proportion of blood cells, among them 100 million of leucocytes (white blood cells) per day on average. Leukocytes act combating and eliminating microorganisms and foreign chemical structures in the body employing a catch (phagocytosis) or antibody production. One of the diseases affecting the bone marrow function is leukemia [1].

Leukemia is a type of cancer that mostly affects the population. The American Cancer Society (ACS) ${ }^{1}$ estimated 61,780 new cases for the year 2019, with approximately 22,840 deaths. This disease has no defined etiology and affects the production of cells by the bone marrow. Over time, diseased cells replace healthy blood cells (white, red blood cells, and platelets), and the individual suffers from problems in transporting oxygen and fighting infections [1]. Among the forms of diagnosis of leukemia, the complete blood count $(\mathrm{CBC})$ and the myelogram are the most used.

There are numerous types of leukemia. However, the most usual classification considers two main characteristics: (1) the cell maturation time, resulting in Acute Leukemia (AL) and Chronic Leukemia (CL), and (2) the leukocyte affected type,

${ }^{1}$ https://www.cancer.org/cancer/leukemia.html

978-1-7281-7539-3/20/\$31.00 @2467422020 IEEE where the two main types of leukocytes are lymphoid and myeloid. Thus, the four main types of leukemia are Acute Lymphoblastic Leukemia (ALL), Acute Myeloid Leukemia (AML), Chronic Lymphocytic Leukemia (CLL), and Chronic Myeloid Leukemia (CML) [1]. While ALs generate an abnormal cells growth in a short time and reach mostly children, CLs tend to reach adults and the elderly, due to the progression in the mature cells production being slower, taking months or even years [1]. Thus, ALL and AML require a diagnosis in the early stages of the disease to provide appropriate treatment. Figure 1 shows examples of blood slide images used in our tests with ALL, AML, and Healthy Blood Slides (HBS).

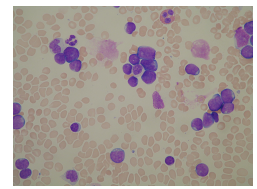

(a)

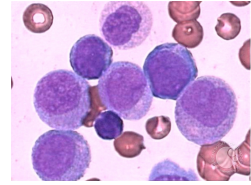

(b)

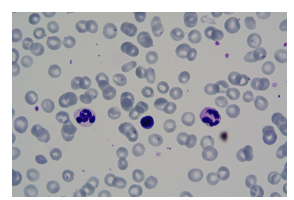

(c)
Figure 1. Examples of images used in this work: (a) ALL example [2], (b) AML example [3], and (c) HBS example [4].

The use of computer systems can assist in fast leukemia diagnosis. Currently, Convolutional Neural Networks (CNNs) are one of the most effective techniques in diagnosing medical images. However, CNNs demand a high computational cost, and in systems with low processing and storage power, this technique becomes difficult to employ [5]. Therefore, in this work, we propose CNN architectures models with residual characteristics to classify blood slides into three classes: ALL, AML, and HBS. When building the model, we evaluated the tradeoff between accuracy and the number of parameters to attain a model that takes up less memory and has performance comparable to the state of the art. We evaluated the proposed model in 16 heterogeneous datasets with 2,415 images, combined with data augmentation techniques. Additionally, we compared the achieved results with the results obtained by the state of the art methods, including based on CCNs.

This paper is organized as follows. Section II presents related works; In Section III, we present the proposed CNN models, the dataset used, the data augmentation technique employed, and the applied evaluation metrics. Sections IV and V present 
the achieved results and a discussion; and finally, we present the conclusion and possibilities of future work in Section VI.

\section{RELATED WORKS}

We carried out a systematic survey of state of the art related to leukemia computer-aided diagnosis. The survey aimed to identify and classify the available works in the literature based on the techniques used, the year of publication, and the relevance.

The survey was realized using three public datasets: Scopus, Web of Science and IEEE Xplore. We used the following search strings: "leukemia classification", "white blood cell classification", and "blood smear leukemia classification". Following this, we selected works published after 2012 in engineering and computer science fields. As a result, we obtained 423 articles. We then analyzed the title and abstract of these, aiming to eliminate repeated documents and those with non-automatic classification methods. Table I presents the works found in the literature, organized according to their purpose.

We organized the selected papers into four approaches using the diagnosis type suggested by the authors. We found studies that performed the diagnosis between images with leukemia and healthy, regardless of the type of leukemia [6]. Some authors differentiated blades of blood with ALL and healthy blades [7]-[14], while other proposals differentiated images with AML and healthy images [15], [16].

The latter approach mentioned above coincides with our proposal and is characterized by the diagnosis into three classes: ALL, AML, and HBS. In Rawat et al. [17], the authors performed the leukocyte nucleus segmentation on 420 images. Then, they analyzed 331 characteristics of each segmented nucleus using a Support Vector Machine (SVM). The work of Laosai and Chamnongthai [18], also, to take into account these categories, they subdivide the types of acute lymphoid leukemia and acute myeloid leukemia. The tests were performed on 500 images, where 150 were of ALL type, 150 of AML type, and 200 of HBS type. According to the authors, the tests showed promising results.

\section{MATERIALS AND Methods}

The purpose of this paper is to present models of CNN architectures to diagnose acute leukemia types in blood slide images. To develop the architectural model proposed in this work, we rely on architectures that recently obtained the best results in leukemia detection, according to the studies found in the literature.

The dataset used in this research hold 2,415 images, which does not represent a large number of images for training a CNN. A solution found to increase the generality of the model, and attack the few cases problem for training the network is the Data Augmentation technique that generates new samples for training.

\section{A. Proposed CNN Models}

Based on state of the art architectures, such as AlexNet [19], CaffeNet [20], and VggNet [21], we initially studied the Acute
Leukemias Recognition Network (Alert Net), a CNN for the acute leukemia classification in blood slides.

Alert Net has five convolutional layers, followed by Batch Normalization and Max Pooling layers. The shallower layers are formed by two fully connected layers, followed by a dropout operation and a softmax layer with three neurons. This model has characteristics existing in sequential architectures presented in state of the art. However, we were searching for a trade-off between the number of parameters and accuracy. Therefore, we proposed a model with 8 million parameters, which is, for example, seven times less than Alex Net.

From the initial model, we carried out an ablation study, to remove or replace layers in Alert Net. Thus, we built two models using technologies implemented in some of the CNNs with the best results in the ImageNet competition. They are ResNet [22] and Xception [23]. We developed the Alert Net with a Residual Layer (Alert Net-R) and the Alert Net with Depthwise Separable Convolutions Layer (Alert Net-X). In Figure 2, it is shown the three models used and illustrated the layer types in each one.
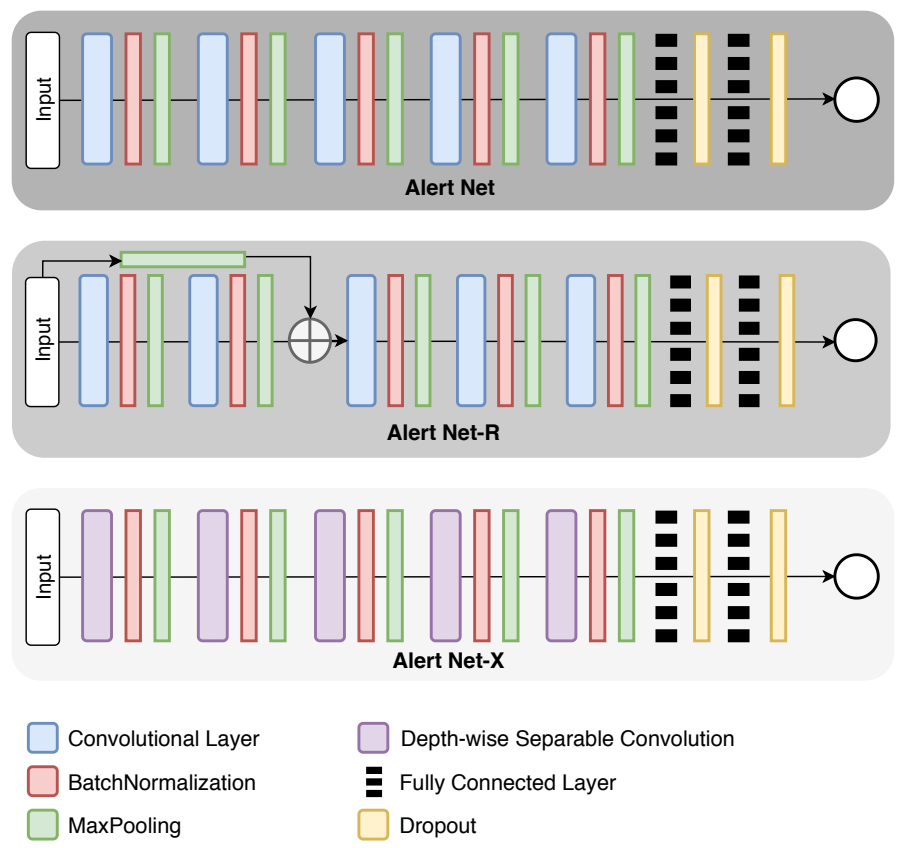

Figure 2. Topologies of the proposed models.

In the Alert Net-R development, we inserted a residual structure similar to ResNet. Initially, we use max pooling after the input layer to resize the original image. The result of this operation is concatenated with the second convolutional layer max pooling result. Therefore, the image to be concatenated does not undergo modifications in the initial convolutional layers. We observed that the residue generated tends to propagate the image's essential characteristics during the training. Studies presented in the literature prove the efficiency of this approach [22].

To develop a model that enables less computationally expensive training, we studied Alert Net-X. The Depth-Wise 
Table I

SUMMARY OF WORKS IDENTIFIED IN THE STATE OF THE ART AS TO: YEAR, DESCRIPTOR(S), CLASSIFIER, NUMBER OF IMAGES USED AND ACCURACY.

\begin{tabular}{lcllll}
\hline Work & Year & Descriptor(s) & Classifier & Images & Accuracy(\%) \\
\hline Leukemia - Healthy & & & & & \\
\hline Vogado et al. [6] & 2018 & Deep Features & SVM & 1268 & 99.76 \\
\hline ALL - Healthy & & & & & \\
\hline Vincent et al. [7] & 2015 & Texture and geometry & MLP & 100 & 97.70 \\
Singhal and Singh [8] & 2016 & Texture & SVM & 260 & 93.80 \\
Thanh et al. [9] & 2017 & Deep Features & CNN & 1188 & 96,60 \\
Shafique et al. [10] & 2018 & Deep Features & CNN & 760 & 99.50 \\
Rehman et al. [11] & 2018 & Deep Features & CNN & 330 & 97.78 \\
Sipes et al. [12] & 2018 & Deep Features & CNN & 388 & 88.00 \\
Pansombut et al. [13] & 2019 & Deep Features & CNN & 363 & 81.74 \\
Ahmed et al. [14] & 2019 & Deep Features & CNN & 2478 & 88.25 \\
\hline AML - Healthy & & & SVM & 50 & 93.50 \\
\hline Madhukar and Agaian [15] & 2012 & Texture & SVM & 90 & 98.00 \\
Goutam and Sailaja [16] & 2015 & Texture & & & 99.50 \\
\hline ALL - AML - Healthy & & & GA-SVM & 420 \\
Rawat et al. [17] & 2017 & Geometrical, color and texture & SVM & 500 & 99.85 \\
Laosai and Chamnongthai [18] & 2018 & Shape, color distribution, texture & & & \\
& & and number of nucleoli & & &
\end{tabular}

Separable Convolution layers were introduced in the Xception architecture and provide greater computational efficiency since the number of operations performed during convolution is reduced. That is, they have less complexity and require less training time than regular convolutional layers.

\section{B. Image Dataset}

The development of a robust methodology to aid in the diagnosis depends on the data used in its validation. The main challenge found in state of the art is related to the acquisition of the datasets since most of them are private. However, we obtained 16 public datasets with 2,415 images for the evaluation of the proposed model. In Table II, the used image datasets are presented according to the addressed classes.

Table II

SUMMARY OF THE USED IMAGE DATASETS

\begin{tabular}{lcccccc}
\hline Dataset & HBS & ALL & AML & Other types & Total & Ref. \\
\hline ALL-IDB 1 & 59 & 49 & - & - & 108 & {$[2]$} \\
ALL-IDB 1 (Crop) & - & 510 & - & - & 510 & {$[2]$} \\
ALL-IDB 2 & 130 & 130 & - & - & 260 & {$[2]$} \\
Leukocytes & 149 & - & - & - & 149 & {$[24]$} \\
CellaVision & 109 & - & - & - & 109 & {$[25]$} \\
Atlas & - & 25 & 40 & 23 & 88 & - \\
Omid et al. 2014 & 154 & - & - & - & 154 & {$[4]$} \\
Omid et al. 2015 & - & - & 27 & - & 27 & {$[26]$} \\
ASH-OK & - & - & 96 & - & 96 & {$[3]$} \\
Bloodline & - & - & 217 & - & 217 & {$[27]$} \\
ONKODIN & - & - & 78 & - & 78 & {$[28]$} \\
CellaVision 2 & 100 & - & - & - & 100 & {$[29]$} \\
JTSC & 300 & - & - & - & 300 & {$[29]$} \\
UFG & 57 & 10 & 27 & 27 & 121 & - \\
PN-ALL Dataset & - & 30 & - & - & 30 & {$[30]$} \\
leukemia-images & - & 40 & 78 & 22 & 140 & - \\
\hline Total of images & $\mathbf{1 0 5 8}$ & $\mathbf{7 9 4}$ & $\mathbf{5 6 3}$ & $\mathbf{7 2}$ & $\mathbf{2 4 8 7}$ & - \\
\hline
\end{tabular}

Among the images listed in Table II, we disregarded those "Other Types" class, since the amount of images in this class does not form a sufficiently representative set. Thus, we used the HBS, ALL, and AML classes in the building of the proposed model. One can note that these classes were built using different datasets, contributing to the creation of a complex set with different resolutions, dyes, approximations, and contrast. Such approach is similar to the one used to obtain microscopic images in daily medical practices [14].

For the proper use of the images under study, we carried out two pre-processing operations. The first was the central clipping considering the smaller side image since $\mathrm{CNN}$ architectures require square inputs. The second operation was to resize the input images to $224 \times 224$ pixels because these are the standard $\mathrm{CNN}$ input dimensions.

In the Bloodline dataset, we observed the existence of 15 rectangular images containing at least two leukocytes. In these images, the clipping of the leukocytes was done manually, since the pre-processing operations would eliminate the region of interest for the classification. The clipped images were added to the dataset, resulting in a total of 217 samples.

\section{Data Augmentation}

Deep neural networks have been successfully applied to Computer Vision tasks such as image classification, object detection, and image segmentation, thanks to the evolution of CNNs. However, these networks rely on a large amount of data to avoid overfitting [31].

Improving generalization of these models is one of the main challenges in the area, but Data Augmentation is a powerful way to overcome this difficulty, Augmented data is expected to represent a more extensive dataset, minimizing the differences between the training and validation sets as well as any future test sets [31].

Usual augmentation operations are rotation in the range of $0^{\circ}$ to $40^{\circ}$, vertical, horizontal, shear, and zoom in the field of 0 to 0.2 , as well as horizontal and vertical flip. One should notice that the nuclei images do not have asymmetry allowing flipping in both directions. The reflection fill operation was applied to replace black pixels resulting from rotation and translation techniques. Finally, we normalized the input image pixels to 
values between 0 (zero) and 1 (one). The augmentation resulted in a dataset 20 times bigger than the original.

\section{Evaluation Metrics}

To analyze the classification results, we computed the confusion matrix. Then, from the elements of this matrix, we calculated the precision $(\mathrm{P})$, recall $(\mathrm{R})$, and accuracy $(\mathrm{A})$.

We also computed the kappa index $(\mathrm{k})$, which is recommended as an appropriate exactitude measure as it can adequately represent the confusion matrix; it takes all elements of the matrix into account, rather than just those on the main diagonal, which occurs when calculating the global classification accuracy [57]. This metric can be calculated as:

$$
\mathrm{k}=\frac{\text { observed }- \text { expected }}{1-\text { expected }}
$$

According to Landis and Koch [32] $\mathrm{k}$ assumes values between 0 (zero) and 1 (one). The result is qualified according to the $\mathrm{k}$ value as follows: $\mathrm{k} \leq 0.2$ : Bad; $0.2<\mathrm{k} \leq 0.4$ : Fair; $0.4<\mathrm{k} \leq 0.6$ : Good; $0.6<\mathrm{k} \leq 0.8$ : Very Good and $\mathrm{k}>0.8$ : Excellent.

The cost function metric (loss) was also used in this work. This function is responsible for saying how far one is from the ideal prediction and, therefore, quantifies the "cost" or "loss" by accepting the prediction generated by the current parameters of the model [33].

\section{Results}

We evaluated the Alert Net, Alert Net-R, and Alert Net$\mathrm{X}$ architectures using $k$-fold cross-validation with $k$ equal to 5 and analyzed the results using state of the art metrics. We also performed experiments with the exclusion of the dropout layer in each model. We chose to perform this analysis since the Batch Normalization layer already works as a regularizer, avoiding overfitting. Removing the dropout layer will not change the essential features for CNNs before the classification stage. In Table III, we present the results obtained by each architecture and corresponding variant without dropout (WD).

Table III

RESULTS OBTAINED BY K-FOLD CROSS VALIDATION FOR ALERT NET.

\begin{tabular}{lccccc}
\hline Model & A (\%) & loss & P (\%) & $\mathrm{R}(\%)$ & $\mathrm{K}$ \\
\hline Alert Net & 95.94 & 0.1186 & 97.37 & 93.45 & 0.9317 \\
Alert Net-WD & 96.72 & 0.0953 & 97.44 & 95.58 & 0.9481 \\
Alert Net-R & 95.85 & 0.1180 & $\mathbf{9 8 . 0 1}$ & 93.32 & 0.9353 \\
Alert Net-RWD & $\mathbf{9 7 . 1 8}$ & $\mathbf{0 . 0 9 4 0}$ & 97.23 & $\mathbf{9 7 . 1 8}$ & $\mathbf{0 . 9 5 6 4}$ \\
Alert Net-X & 94.76 & 0.1575 & 94.94 & 94.77 & 0.9194 \\
Alert Net-XWD & 95.53 & 0.1399 & 95.72 & 95.20 & 0.9324 \\
\hline
\end{tabular}

From the results presented in Table III, we found that Alert Net-RWD achieved the best results in terms of Accuracy (97.18\%), Recall (97.18\%), and kappa (0.9564), while Alert Net-R obtained the highest Precision rate (98.01\%). We also highlight the results obtained by the Alert Net without dropout.

The results in Table III allow us to infer that the dropout layer removal promoted in achieving better results. The dropout is a regularization technique, and its use reduces the generalization capacity of the model. To deal with this, it would be necessary to increase the model size because typically, the error in the validation dataset is much smaller when using dropout, but with accounting larger models cost more training iterations. When the training dataset is small, the use of the dropout becomes less effective.

\section{Discussion}

We carry out a comparison among the Alert Net-RWD results and the ones obtained by works of literature that address the same problem. From Table IV, one can realize that the works in the literature presented higher accuracy values than Alert NetRWD. However, the number of images used in those studies is at least four times less than the number of images used in this work. Another critical point is that only the current research used more than one image dataset. This characteristic leads to a greater diversity in the training data, which leads to the achievement of a robust method for different input image types.

Table IV

COMPARISON AMONG THE RESULTS OBTAINED BY THE PROPOSED AGAINST THE ONES OBTAINED BY RELATED METHODS.

\begin{tabular}{cccc}
\hline Method & Descritors & $\begin{array}{c}\text { N. of } \\
\text { images }\end{array}$ & Accuracy \\
\hline Rawat et al. [17] & Geometrical, color and texture & 420 & $99.50 \%$ \\
\hline $\begin{array}{c}\text { Laosai and } \\
\text { Chamnongthai [18] }\end{array}$ & $\begin{array}{c}\text { Shape, color, Texture } \\
\text { and number of nucleoli }\end{array}$ & 500 & $99.85 \%$ \\
\hline Proposed method & Deep Features & 2415 & $97.18 \%$ \\
\hline
\end{tabular}

For the best trade-off between network accuracy and complexity, we evaluated and compared Alert Net-RWD against the CNNs: ResNet50, InceptionV3, and Xception. These CNNs presented excellent results in the ImageNet competition. Also, they have a relatively low number of parameters when compared to other sequential architectures. We applied fine-tuning techniques [34], which consists of using a pre-trained architecture to carry out the transfer learning. Thus, training is only conducted in selected layers and with lower learning rates.

The most commonly used fine-tuning technique used in the literature is Shallow fine-tuning (SFT) [34]. In SFT, the initial layers are frozen, which tends to decrease the complexity during the CNN training, and only the final layers are retrained. These layers have specific characteristics related to the used dataset. Table V presents the results from obtained applying the SFT technique with the CNNs found in the literature.

From Table V, one can confirm the Alert Net-RWD superiority, by achiving kappa values considered excellent, while the other CNNs did not obtain satisfactory performances. The ResNet50 architecture, for example, got a kappa value equal to 0 (zero), which means that this CNN classified all images in one class. Besides having achieved the best performance in terms of classification, Alert Net-RWD generates a smaller file using fewer parameters.

Some authors claim that SFT does not perform well when the target domain differs from one used to pre-train the weights [35]. For example, natural photographic images from ImageNet 
Table V

Results obtained With Shallow Fine Tuning.

\begin{tabular}{lccccccc}
\hline Model & $\mathrm{A}(\%)$ & loss & $\mathrm{P}(\%)$ & $\mathrm{R}(\%)$ & $\mathrm{K}$ & Num. param & File size \\
\hline ResNet50 & $41.61 \pm 4.86$ & $1.2667 \pm 0.11$ & $18.50 \pm 1.48$ & $41.61 \pm 4.86$ & 0 & $24,638,339$ & $94 \mathrm{mb}$ \\
InceptionV3 & $64.30 \pm 2.09$ & $0.8231 \pm 0.03$ & $65.25 \pm 1.73$ & $64.30 \pm 2.09$ & $0.4501 \pm 0.03$ & $22,853,411$ & $109 \mathrm{mb}$ \\
Xception & $72.00 \pm 3.48$ & $0.6913 \pm 0.02$ & $71.43 \pm 3.64$ & $72.084 \pm 3.47$ & $0.5601 \pm 0.05$ & $22,910,480$ & $90 \mathrm{mb}$ \\
Alert Net-RWD & $\mathbf{9 7 . 1 8} \pm 0.61$ & $\mathbf{0 . 0 9 4 0} \pm 0.03$ & $\mathbf{9 7 . 2 3} \pm 0.61$ & $\mathbf{9 7 . 1 8} \pm 0.60$ & $\mathbf{0 . 9 5 6 4} \pm 0.0094$ & $\mathbf{8 , 7 9 2 , 9 6 3}$ & $\mathbf{6 8 m b}$ \\
\hline
\end{tabular}

belong to a different domain when compared to blood smear images. In these situations, it is better to apply Deeply FineTuning (DFT). The DFT approach allows training the entire network. However, it requires a higher computational cost and a more considerable amount of data. Table VI, presents the results obtained by applying DFT with the CNNs found in the literature.

Comparing Tables $\mathrm{V}$ and VI, one can realize that the use of DFT resulted in a substantial performance gain of the pretrained CNNs. With DFT, ResNet50 overcome Alert Net-RWD with the best results in terms of accuracy (97.80\%), precision (97.81\%), recall $(97.80 \%)$, and kappa (0.9660).

We performed a statistical evaluation using the student $\mathrm{T}$ test with a significance level of $5 \%$ and found that the results achieved by Alert Net-RWD and ResNet50 are statistically equivalent. Therefore, one can conclude that Alert Net-RWD, although less complex (it has about one-third of the parameters relative to other $\mathrm{CNNs}$ ), achieves results comparable to pretrained architectures. Analyzing the file size generated from the training, we observed that Alert Net-RWD is more attractive for use on mobile devices. These devices can have a crucial role in the disease diagnosis in isolated regions, for example.

Figure 3 shows examples of the Alert Net-RWD activation maps for the three classes. It is possible to identify which regions are used to differentiate healthy images from those with acute leukemia (lymphoid or myeloid).
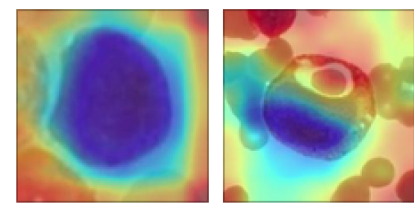

(a)
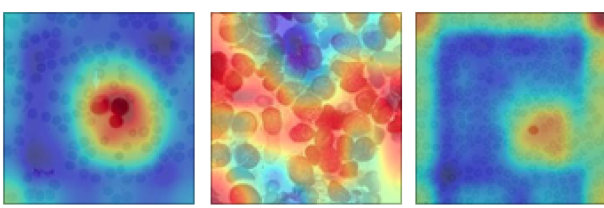

(b)

Figure 3. Examples of activation maps for blood slides, (a) images with one leukocyte, (b) images with various leukocytes. The first column are images of the ALL class; the second column are images of the AML class and the third of the HBS class.

The number of leukocytes may vary depending on the input image. We see in Figure 3 that Alert Net-RWD generates different activation map patterns for each of these situations. Also, as it is trained in different databases, the proposed model can adapt to different characteristics, it is possible to observe that the maps differ from one base to another. However, CNN activates different regions for each class. For example, in figure 3.a, of the ALL class, the leukocyte turned predominantly blue. This pattern changes in the other classes, becoming mostly red in the HBS class.

\section{CONCLUSION}

The conducted systematic survey showed that many researchers had focused their efforts on the Computer-Aided Diagnostic systems field, where the automatic diagnosis of leukemia can be found.

In this work, we developed Alert Net-RWD, a CNN model for the automated diagnosis of acute lymphoid and acute myeloid leukemia. The achieved results are promising, and the number of parameters used in Alert Net-RWD is inferior to the ones of other architectures found in the literature. Also, the proposed model has a smaller file size, which makes it more attractive to be used in applications on mobile devices.

The bibliographical survey also showed that authors usually use only a single private dataset to evaluate their proposals. However, this situation does not represent a real environment for the application of this type of system. Therefore, we evaluated our approach in an high heterogeneous image dataset.

For future approaches, the proposed model needs to be applied to a more significant number of images. Moreover, in addition to differentiation of the three classes proposed in this work, a distinction will also be made between the images that have Chronic Lymphocytic Leukemia and Chronic Myeloid Leukemia.

\section{ACKNOWLEDGEMENTS}

This study was partially founded by the Coordenação de Aperfeiçoamento de Pessoal de Nível Superior - Brasil (CAPES) - Finance Code 001 and Fundação de Amparo à Pesquisa do Piaui (FAPEPI). We gratefully acknowledge the support of NVIDIA Corporation with the donation of the Titan $\mathrm{Xp} \mathrm{GPU} \mathrm{used} \mathrm{in} \mathrm{this} \mathrm{research.}$

\section{REFERENCES}

[1] G. S. Travlos, "Normal structure, function, and histology of the bone marrow," Toxicologic Pathology, vol. 34, no. 5, pp. 548-565, 2006. 
Table VI

RESUlts obTained With DEEPLy Fine Tuning.

\begin{tabular}{lccccccc}
\hline Model & $\mathrm{A}(\%)$ & loss & $\mathrm{P}(\%)$ & $\mathrm{R}(\%)$ & $\mathrm{K}$ & Num. param & File size \\
\hline ResNet50 & $\mathbf{9 7 . 8 0} \pm 0.59$ & $\mathbf{0 . 0 6 7 3} \pm 0.01$ & $\mathbf{9 7 . 8 1} \pm 0.59$ & $\mathbf{9 7 . 8 0} \pm 0.59$ & $\mathbf{0 . 9 6 6 0} \pm 0.0093$ & $24,638,339$ & $192 \mathrm{mb}$ \\
InceptionV3 & $97.02 \pm 0.61$ & $0.0921 \pm 0.02$ & $97.04 \pm 0.59$ & $97.01 \pm 0.61$ & $0.9537 \pm 0.0095$ & $23,851,784$ & $179 \mathrm{mb}$ \\
Xception & $95.98 \pm 0.55$ & $0.1056 \pm 0.01$ & $96.08 \pm 0.58$ & $95.98 \pm 0.56$ & $0.93782 \pm 0.0086$ & $22,910,480$ & $171 \mathrm{mb}$ \\
Alert Net-RWD & $97.18 \pm 0.61$ & $0.0940 \pm 0.03$ & $97.23 \pm 0.61$ & $97.18 \pm 0.60$ & $0.9564 \pm 0.0094$ & $\mathbf{8 , 7 9 2 , 9 6 3}$ & $\mathbf{6 8 m b}$ \\
\hline
\end{tabular}

[2] R. D. Labati, V. Piuri, and F. Scotti, "All-idb: The acute lymphoblastic leukemia image database for image processing." in IEEE International Conference on Image Processing, 2011, pp. 2045-2048.

[3] M. Madhukar, S. Agaian, and A. T. Chronopoulos, "Automated screening system for acute myelogenous leukemia detection in blood microscopic images," IEEE Systems Journal, vol. 8, no. 3, pp. 995-1004, 2014.

[4] O. Sarrafzadeh, H. Rabbani, A. Talebi, and H. U. Banaem, "Selection of the best features for leukocytes classification in blood smear microscopic images," in SPIE Medical Imaging, vol. 9041, 2014.

[5] M. I. Razzak, S. Naz, and A. Zaib, "Deep learning for medical image processing: Overview, challenges and the future," in Classification in BioApps. Springer, 2018, pp. 323-350.

[6] L. H. S. Vogado, R. M. S. Veras, F. H. D. Araújo, R. R. V. e Silva and K. R. T. Aires, "Leukemia diagnosis in blood slides using transfer learning in cnns and SVM for classification," Engineering Applications of Artificial Intelligence, vol. 72, pp. 415-422, 2018.

[7] I. Vincent, K.-R. Kwon, S.-H. Lee, and K.-S. Moon, "Acute lymphoid leukemia classification using two-step neural network classifier," Frontiers of Computer Vision, pp. 1-4, 2015

[8] V. Singhal and P. Singh, Texture Features for the Detection of Acute Lymphoblastic Leukemia. Singapore: Springer Singapore, 2016, pp. 535 543.

[9] T. T. P. Thanh, C. Vununu, S. Atoev, S.-H. Lee, and K.-R. Kwon, "Leukemia blood cell image classification using convolutional neural network," International Journal of Computer Theory and Engineering, vol. 10, no. 2, pp. 54-58, 2018.

[10] S. Shafique and S. Tehsin, "Acute lymphoblastic leukemia detection and classification of its subtypes using pretrained deep convolutional neural networks," Technology in Cancer Research and Treatment, vol. 17, pp. 1-7, september 2018 .

[11] A. Rehman, N. Abbas, T. Saba, S. I. ur Rahman, Z. Mehmood, and H. Kolivand, "Classification of acute lymphoblastic leukemia using deep learning," Microscopy Research and Technique, pp. 1-8, October 2018.

[12] R. Sipes and D. Li, "Using convolutional neural networks for automated fine grained image classification of acute lymphoblastic leukemia," in International Conference on Computational Intelligence and Applications. IEEE, 2018, pp. 157-161.

[13] T. Pansombut, S. Wikaisuksakul, K. Khongkraphan, and A. Phon-on, "Convolutional neural networks for recognition of lymphoblast cell images," Computational Intelligence and Neuroscience, vol. 2019, pp. $1-12,2019$.

[14] N. Ahmed, A. Yigit, Z. Isik, and A. Alpkocak, "Identification of leukemia subtypes from microscopic images using convolutional neural network,' Diagnostics, vol. 9, no. 3, p. 104, 2019.

[15] M. Madhukar, S. Agaian, and A. T. Chronopoulos, "Deterministic model for acute myelogenous leukemia classification," in IEEE International Conference on Systems, Man, and Cybernetics. IEEE, 2012, pp. 433 438.

[16] D. Goutam and S. Sailaja, "Classification of acute myelogenous leukemia in blood microscopic images using supervised classifier," in 2015 IEEE International Conference on Engineering and Technology (ICETECH). IEEE, 2015, pp. 1-5.

[17] J. Rawat, A. Singh, B. HS, J. Virmani, and J. S. Devgun, "Computer assisted classification framework for prediction of acute lymphoblastic and acute myeloblastic leukemia," Biocybernetics and Biomedical Engineering, vol. 37, no. 4, pp. 637 - 654, 2017.

[18] J. Laosai and K. Chamnongthai, "Classification of acute leukemia using medical-knowledge-based morphology and cd marker," Biomedical Signal Processing and Control, vol. 44, pp. 127-137, 2018.
[19] A. Krizhevsky, I. Sutskever, and G. E. Hinton, "Imagenet classification with deep convolutional neural networks," in Advances in neural information processing systems, 2012, pp. 1097-1105.

[20] Y. Jia, E. Shelhamer, J. Donahue, S. Karayev, J. Long, R. Girshick, S. Guadarrama, and T. Darrell, "Caffe: Convolutional architecture for fast feature embedding," in ACM International Conference on Multimedia. ACM, 2014, pp. 675-678.

[21] K. Chatfield, K. Simonyan, A. Vedaldi, and A. Zisserman, "Return of the devil in the details: Delving deep into convolutional nets," arXiv preprint arXiv:1405.3531, 2014.

[22] K. He, X. Zhang, S. Ren, and J. Sun, "Deep residual learning for image recognition," in IEEE Conference on Computer Vision and Pattern Recognition. IEEE, 2016, pp. 770-778.

[23] F. Chollet, "Xception: Deep learning with depthwise separable convolutions," in IEEE Conference on Computer Vision and Pattern Recognition, 2017, pp. 1800-1807.

[24] O. Sarrafzadeh and A. M. Dehnavi, "Nucleus and cytoplasm segmentation in microscopic images using $\mathrm{k}$ means clustering and region growing," Advanced Biomedical Research, pp. 79-87, December 2015.

[25] M. Rollins-Raval, J. Raval, and L. Contis, "Experience with cellavision dm96 for peripheral blood differentials in a large multi-center academic hospital system," Journal of Pathology Informatics, vol. 3, no. 29, pp. $1-9,2012$.

[26] O. Sarrafzadeh, H. Rabbani, A. M. Dehnavi, and A. Talebi, "Detecting different sub-types of acute myelogenous leukemia using dictionary learning and sparse representation." in IEEE International Conference on Image Processing. IEEE, 2015, pp. 3339-3343.

[27] A. M. P. G. Vale, A. M. G. Guerreiro, A. D. D. Neto, G. B. Cavalvanti Junior, V. C. L. T. de Sá Leitão, and A. M. Martins, "Automatic segmentation and classification of blood components in microscopic images using a fuzzy approach,” Revista Brasileira de Engenharia Biomédica, vol. 30, pp. 341-354, 2014.

[28] J. Böhm, "Pathologie-websites im world wide web," Der Pathologe, vol. 29, no. 3, pp. 231-242, 2008.

[29] X. Zheng, Y. Wang, G. Wang, and Z. Chen, "Fast and robust segmentation of white blood cell images by self-supervised learning," Micron, vol. 107, pp. 55-71, 2018.

[30] R. Duggal, A. Gupta, R. Gupta, and P. Mallick, "Sd-layer: Stain deconvolutional layer for cnns in medical microscopic imaging," in Medical Image Computing and Computer Assisted Intervention, M. Descoteaux, L. Maier-Hein, A. Franz, P. Jannin, D. L. Collins, and S. Duchesne, Eds. Springer, 2017, pp. 435-443.

[31] C. Shorten and T. M. Khoshgoftaar, "A survey on image data augmentation for deep learning," Journal of Big Data, vol. 6, no. 1, p. 60, July 2019.

[32] J. R. Landis and G. G. Koch, "The measurement of observer agreement for categorical data," Biometrics, pp. 159-174, 1977.

[33] K. Janocha and W. M. Czarnecki, "On loss functions for deep neural networks in classification," arXiv:1702.05659, 2017.

[34] K. Pasupa and W. Sunhem, "A comparison between shallow and deep architecture classifiers on small dataset," in International Conference on Information Technology and Electrical Engineering. IEEE, 2016, pp. $1-6$.

[35] M. Izadyyazdanabadi, E. Belykh, M. Mooney, N. Martirosyan, J. Eschbacher, P. Nakaji, M. Preul, and Y. Yang, "Convolutional neural networks: Ensemble modeling, fine-tuning and unsupervised semantic localization for neurosurgical cle images," Journal of Visual Communication and Image Representation, vol. 54, pp. 10-20, 72018. 